\title{
Some new structural and electronic characteristics of quasicrystals
}

\author{
O N SRIVASTAVA*, R S TIWARI, N P LALLA ${ }^{\dagger}, \mathbf{R}$ K MANDAL ${ }^{\ddagger}$ and M A SHAZ \\ Physics Department, Banaras Hindu University, Varanasi 221005 , India \\ ${ }^{\dagger}$ IUC, DAEF, Khandwa Road, Indore, India \\ ${ }^{\ddagger}$ Metallurgy Department, Banaras Hindu University, Varanasi 221005 , India
}

\begin{abstract}
The quasicrystals being based on quasiperiodic order other than crystal like periodic translational order and embodying self similarity, present unique condensed matter phases. In addition to their curious structural characteristics the paucity of translational periodicity leads to drastic deviations in their electronic behaviour as compared to crystalline counterparts. This paper describes and discusses some new developments in regard to structural and electronic aspects of quasicrystalline materials. In regard to the structural aspects, two comparatively newer features will be described. One of them relates to the observation of variable strain approximants (VSA) first found in $\mathrm{Ti}_{68} \mathrm{Fe}_{26} \mathrm{NiSi}_{5}$, qc alloys; the other relates to the structure of decagonal phases. The variable strain approximants correspond to qc phases exhibiting variable strain for the different diffraction spots for the same reciprocal lattice row (possessing linear shifts). The VSA is thought to result from variable phason strain mode locking; this in contrast to RAS which results due to linear phason mode locking. The results obtained in our laboratory on VSA will be described and discussed. Another interesting structural feature emanating in the last few years relate to the development of structural models for the decagonal phases which have nearly answered the question 'Where are the atoms?' for this qc variant. High resolution electron microscopy has revealed the existence of two types of atom cluster columns with a diameter of $20 \AA$; a pentagonal cluster column and a decagonal one. The decagonal quasicrystals can be classified into three types of structures according to the space groups and symmetries and arrangements of the cluster columns. These three deca structures have been typified by: deca $\mathrm{Al}-\mathrm{Co}-\mathrm{Cu}$, deca $\mathrm{Al}-\mathrm{Mn}$ and deca Al-Fe-Pd types. Some basic features on the structures of decagonal phases as obtained recently will be outlined.

Since materials owe their practical importance due to their physical behaviours, assessment of qc materials from this point of view is of imperative importance. However, the real physics/science governing properties for $q \mathrm{c}$ is not well understood as yet. For example, the fundamental property relating to electronic conductivity and its temperature variation has been attempted to become explicable based on (a) taking qc as disordered materials, (b) assuming qc as hierarchy of clusters and (c) bringing in new concepts governing the wave functions of electrons (critical wave functions) and some other models. However, the same results are not universally reported/reproduced by various workers. Thus the variation of electronic conductivities with temperature of $\mathrm{Al}_{65} \mathrm{Cu}_{20} \mathrm{Ru}_{15}, \mathrm{Al}_{70} \mathrm{Pd}_{20} \mathrm{Re}_{10}$ and other stable quasicrystals have found varied interpretations e.g. based on QIEs and power law temperature variation. Some results on $\sigma-T$ and related characteristics for stable qe crystals obtained by us and also by other workers will be analysed in terms of feasible transport mechanisms.
\end{abstract}

Keywords. Quasicrystals; structure and microstructure; electron transport.

\section{Introduction}

The two important characteristics of quasicrystalline (qc) materials which appear to be of imperative importance relates to the structural and physical (e.g. electronic) property.

As regards to the structural property, ever since the seminal finding of icosahedral quasicrystal (IQC) in rapidly solidified alloy of $\mathrm{Al}_{86} \mathrm{Mn}_{14}$ (Shechtman et al 1984), a surfeit of structure related to it is reported. Based on elemental composition, qc can be classified broadly into two categories: (i) aluminium ( $\mathrm{Al}$ ) bearing

*Author for correspondence qc system and (ii) titanium/zirconium (Ti/Zr) bearing qc system. A curious structural variant of IQC are rational approximant structures (RAS). It has been argued that a series of periodic structures can be generated, by sectioning the higher dimensional crystal, lying in pseudo space by the physical space whose orientations are fixed by replacing the golden mean $(\tau)$, with some rational value.

In the case of RAS if the fixation of physical space occur with ratio in between the approximant of $\tau$, these are known as non-Fibonacci RAS (Mukhopadhya et al 1993; Zhang et al 1994).

RAS are thought to be resulting from mode locking of the linear phason strain $(\gamma)$. In this the strain of the different spot of reciprocal pattern are same. 


\section{Structural properties: VSA and decagonal structure}

\subsection{Variable strain approximants}

However, in the present investigation (Shaz et al 1997) embodying $\mathrm{Ti}_{68} \mathrm{Fe}_{26} \mathrm{Ni}_{1} \mathrm{Si}_{5}$ quasicrystals, a new type of approximant viz. variable strain approximant (VSA) has been found.

In this case the $(\gamma)$ for the different spots in the same reciprocal rows are different e.g. along the true two-fold direction. To illustrate this point we reproduce the fivefold diffraction pattern from IQC in figure 1 and pseudo five-fold diffraction pattern from the different sectors of the same grain having variable phason strain, yielding different pseudo five-fold diffraction patterns from different sectors shown in figure 2 . Even though these patterns look similar, careful observation reveal that they are different in detail e.g. the five-fold (or ten-fold due to Freidel's law) spot closest to transmitted beam has different sense of distortion.

This is further clarified by two-fold diffraction pattern shown in figure 3 . Here again the $\gamma$ value for different spots as shown by arrows are different.

It may be mentioned that strain $(\gamma)$ are calculated using the standard expression shown here.

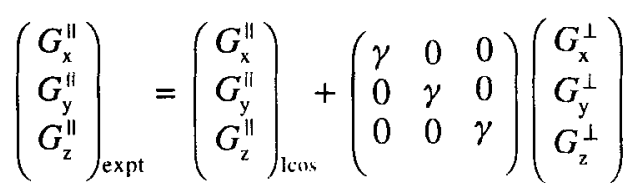
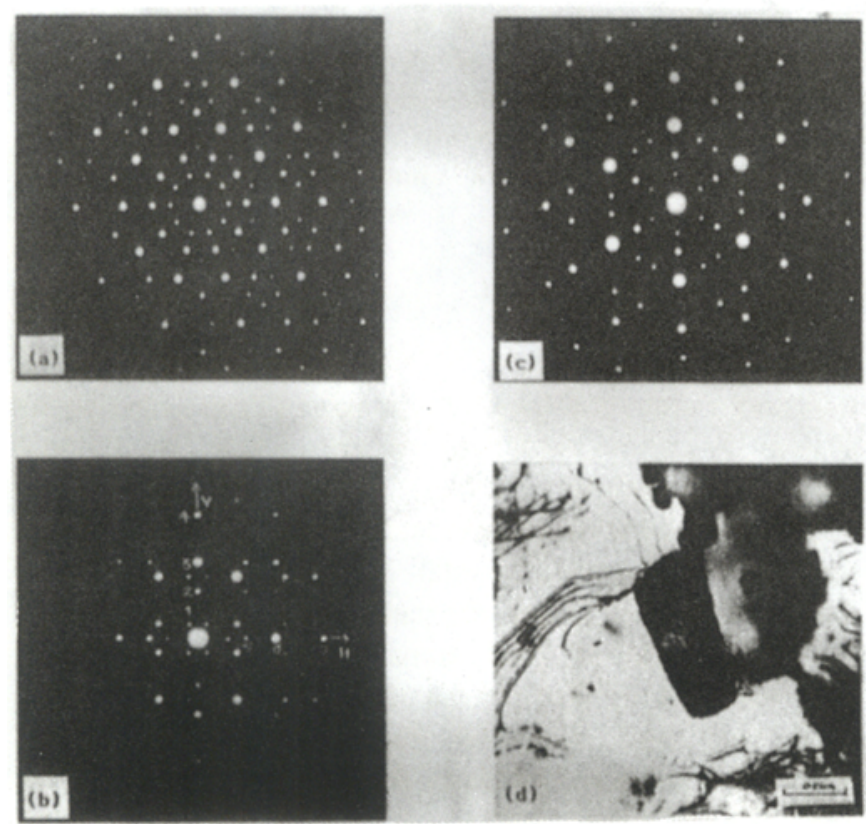

Figure 1. (a) Five-fold, (b) two-fold, and (c) three-fold SAD patterns taken from the region whose microstructure is shown in (d). These SAD patterns reveal the icosahedral symmetry of the phase present in that particular region.
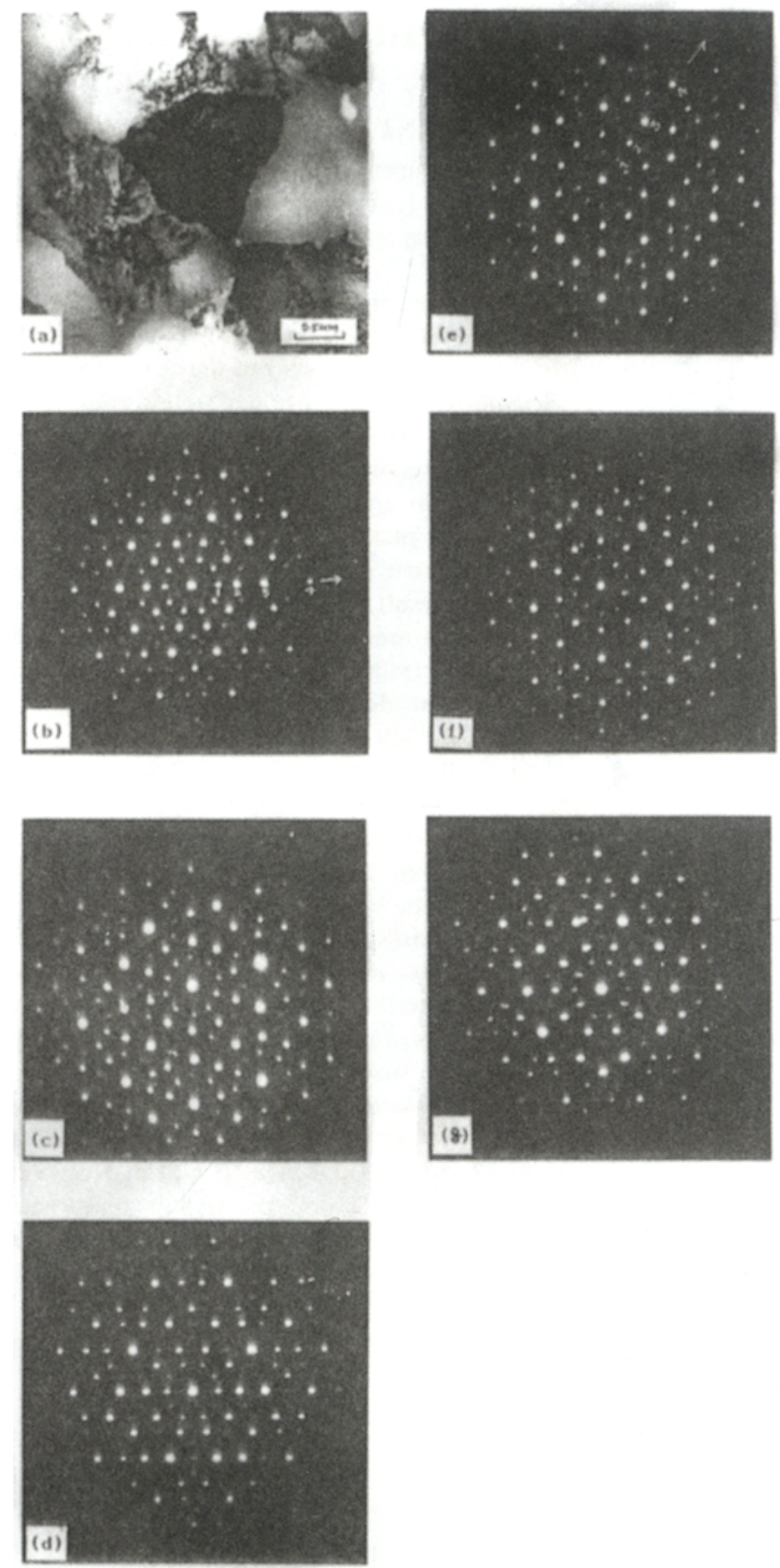

Figure 2. (a) Microstructure revealing the sectorial contrast emerging radially from the central region of the grain, (b) SAD pattern from close to the central region, (c)-(g) represent the SAD patterns from the different sectors of the same grain. These represent the pseudo five-fold (or broken) symmetry. The difference between various patterns can be observed by comparing the directions of the shifts of spots. In each pattern there is only one direction i.e. [100]-type direction, along which the spots suffer only linear shifts. This direction has been indicated by an arrow in (b) and (e) whose spots along this direction have been used for calculation of the $\gamma$ value of the phason strain matrix. 
where $G_{\mathrm{x}}^{\|} G_{\mathrm{y}}^{\|} G_{\mathrm{z}}^{\|}$and $G_{\mathrm{x}}^{\perp} G_{\mathrm{y}}^{\perp} G_{\mathrm{z}}^{\perp}$ are respectively, physical and pseudo space Cartesian components of any 6D reciprocal lattice vectors, $\gamma$ is the coefficient of phason matrix.

The details of $G^{\|}$and $G^{\perp}$ and $\gamma$ values are listed in tables $1 \mathrm{a}, \mathrm{b}$ and $\mathrm{c}$.

It should be pointed out that the occurrence of VSA should be general and not specific to the present elemental composition. Since the logic of presence of VSA should be a naturally occurring feature of IQC phase.

\subsection{Decagonal structures}

Yet another curious structural variant as observed in last few years correspond to unravelling of the structure of decagonal phases. It has now become known that deca-

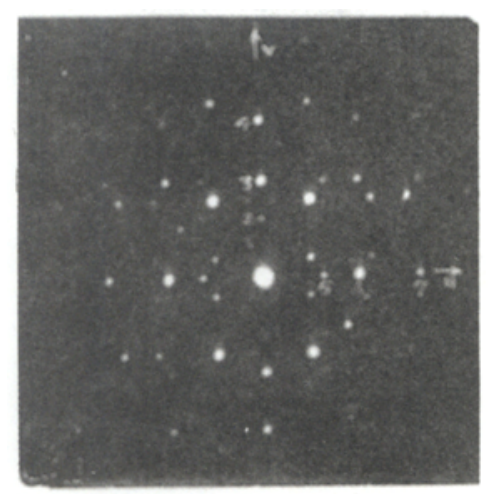

Figure 3. The vertically and horizontally oriented proper twofold direction along which the spots have been used for measurement for the $\gamma$ value of the phason strain matrix is shown.

Table 1a. $G^{11}$ and $G^{\perp}$ values of IQC.

\begin{tabular}{llll}
\hline $\begin{array}{l}\text { Spot } \\
\text { number }\end{array}$ & $\begin{array}{c}\text { Indices of } \\
\text { reflection }\end{array}$ & \multicolumn{1}{c}{$G^{\text {II }}$} & \multicolumn{1}{c}{$G^{\perp}$} \\
\hline 1 & 100010 & 1.102 & +0.680 \\
2 & 101011 & 1.799 & -0.419 \\
3 & 201021 & 2.902 & +0.261 \\
4 & 302032 & 4.717 & -0.159 \\
5 & 011101 & 1.799 & -0.4192 \\
6 & 021201 & 2.9017 & +0.2606 \\
7 & 032302 & 4.7167 & -0.1586 \\
\hline
\end{tabular}

gonal qc phases are built from quasiperiodically packed columnar clusters with symmetry $10_{5} / \mathrm{mmc}$ or $10_{5} \mathrm{mc}$. The clusters are periodic along their ten-fold axis and consist of stacking of $2,4,6,8,12$ or 18 flat or puckered atomic layer leading to translation period of $\approx 4 \AA, \approx 8 \AA, \approx 12 \AA, \approx 16 \AA, \approx 24 \AA$ and $\approx 36 \AA$, respectively. It has been possible to describe the structure based on three basic decagonal structure types. These are (i) deca $\mathrm{Al}-\mathrm{Co}-\mathrm{Cu}$ type, (ii) deca Al-Mn type and (iii) deca $\mathrm{Al}-\mathrm{Fe}-\mathrm{Pd}$ type. Here in order to elucidate the observed structural detail we will describe one of the above three types viz. deca $\mathrm{Al}-\mathrm{Co}-\mathrm{Cu}$ type following details and description by Steurer (1994) and Tsuda et al (1997). Some salient features of this deca type phase are as follows.

(I) Two-layer structure with translation period $\approx 4 \AA$. The planar layers are stacked with sequence $\mathrm{Aa}$ ( $\mathrm{a}^{\prime}$ means a layer rotated around $36^{\circ}$ under the action of the $10^{5}$-screw axis). The formation of a network of icosagonal rings of pentagonal and rectangular structure motifs may act as weak matching rule stabilizing the quasiperiodic tiling (figure 4a). Monoclinic $\mathrm{Al}_{13} \mathrm{Co}_{4}$ $\left(\mathrm{Al}_{13} \mathrm{Fe}_{4}\right.$ type) is a closely related approximant structure with the same pentagon-rectangle strips which are arranged, however, in wavy bands instead of icosagons (figure $4 b$ ).

(II) There are two hyperatoms per asymmetric unit on Wyckoff position (b): one with $p=2$ consisting mainly of transition metal (marked 1), and one with $p=4$ (marked 2) consisting of $\mathrm{Al}$ atoms (figure 4c).

(III) Shape and chemical composition of the hyperatoms are roughly compatible with tiling models like a nonperfect Penrose tiling or a binary tiling model.

Table 1c. $\gamma$ values for vertical two-fold and horizontal two-fold directions.

\begin{tabular}{lccccrr}
\hline & \multicolumn{2}{c}{ Vertical direction } & & \multicolumn{2}{c}{ Horizontal direction } \\
\cline { 2 - 3 } \cline { 5 - 6 } $\begin{array}{l}\text { Spot } \\
\text { number }\end{array}$ & $G_{\text {expt }}^{\text {॥ }}$ & $\gamma$ & $\begin{array}{c}\text { Spot } \\
\text { number }\end{array}$ & $G_{\text {expt }}^{\text {II }}$ & $\gamma$ \\
\hline 1 & 1.051 & -0.074 & 5 & 1.752 & 0.111 \\
2 & 1.791 & +0.018 & 6 & 2.843 & -0.224 \\
3 & 2.843 & -0.224 & 7 & 4.558 & -0.100 \\
4 & 4.551 & +1.044 & & & \\
\hline
\end{tabular}

Table 1b. $G_{\text {expt }}^{\mathrm{II}}$ and calculated $\gamma$ values of VSAs.

\begin{tabular}{lcccccccccccc}
\hline & \multicolumn{2}{c}{ Fig. 2(b) } & \multicolumn{2}{c}{ Figs $2(\mathrm{c}-\mathrm{g})$} & \multicolumn{2}{c}{ Fig. 4(a) } & \multicolumn{2}{c}{ Fig. 4(b) } & \multicolumn{2}{c}{ Fig. 4(c) } \\
\cline { 2 - 11 } $\begin{array}{l}\text { Spot } \\
\text { number }\end{array}$ & $G_{\text {expt }}^{\prime \prime}$ & $\gamma$ & $G_{\text {expt }}^{\prime \prime}$ & $\gamma$ & $G_{\text {expt }}^{\text {II }}$ & $\gamma$ & $G_{\text {expt }}^{\text {II }}$ & $\gamma$ & \multicolumn{1}{c}{$G_{\text {expt }}^{\text {II }}$} & $\gamma$ \\
\hline 1 & 1.012 & -0.131 & 0.993 & -0.160 & 1.051 & 0.074 & 1.012 & -0.131 & 0.973 & -0.189 \\
2 & 1.870 & -0.168 & 1.791 & +0.018 & 1.791 & +0.018 & 1.811 & -0.028 & 1.811 & -0.027 \\
3 & 2.902 & -0.001 & 2.882 & -0.075 & 2.843 & -0.224 & 2.823 & +0.298 & 2.765 & -0.523 \\
4 & 4.732 & -0.101 & 4.693 & +0.147 & 4.551 & +1.044 & 4.596 & +0.761 & & \\
\hline
\end{tabular}


(IV) Columnar clusters $\left(\phi=20 \AA\right.$ ) with symmetry $10_{5} / \mathrm{mmc}$ are the basic structural units. Their cross sections can be described as parts of a Penrose tiling. The unit rhombus with edge lengths $a_{\mathrm{r}}=2.5 \AA$ are decorated by atoms on the vertices and partially also on the diagonals (figure 5). (V) The global structure can be described as a rhombic tiling with unit tiles of edge length $a_{\mathrm{r}}=20 \AA$ (equivalent to the distance between two $=20 \AA$ columnar clusters), decorated by the columnar clusters at the vertices and at one position on the long diagonal of the fat rhomb (figure $4 \mathrm{~d}$ and 5 ).

\section{Physical properties: Electronic behaviour of quasicrystals}

The qc materials are alloys, hence are expected to show metallic conductivity but actually they are found to exhibit behaviour closer to semiconductor/insulator. In fact the higher the perfection of the qc material, lower is the conductivity they exhibit (Pierce et al 1992; Lalla et al 1995; Wang et al 1997) (for $\mathrm{Al}-\mathrm{Cu}-\mathrm{Ru}$ the conductivity at $300 \mathrm{~K}$ is as low as $6 \times 10^{-5} \Omega^{-1} \mathrm{~cm}^{-1}$, for Al-Pd-Re it is still smaller by about two orders of magnitude). This unusual feature and the related aspects have motivated a surfeit of studies on the electronic behaviour of quasicrystals particularly for the stable qc materials such as $\mathrm{Al}-\mathrm{Cu}-\mathrm{Ru}, \mathrm{Al}-\mathrm{Cu}-\mathrm{Fe}, \mathrm{Al}-\mathrm{Pd}-\mathrm{Re}$ etc.

The electronic behaviour of qc materials is not well understood at present. The difficulty partly arises due to unknown details of the electronic structure of quasicrystals and partly to the ambiguous characterization of qc for perfection (phason disorder). Often the qc studies even after considerable refinement contain phason disorder capable of influencing electron transport.

Several models/mechanisms have been proposed to make the electronic behaviours (e.g. such simplest behaviour is manifest in $\sigma-T$ without and with applied
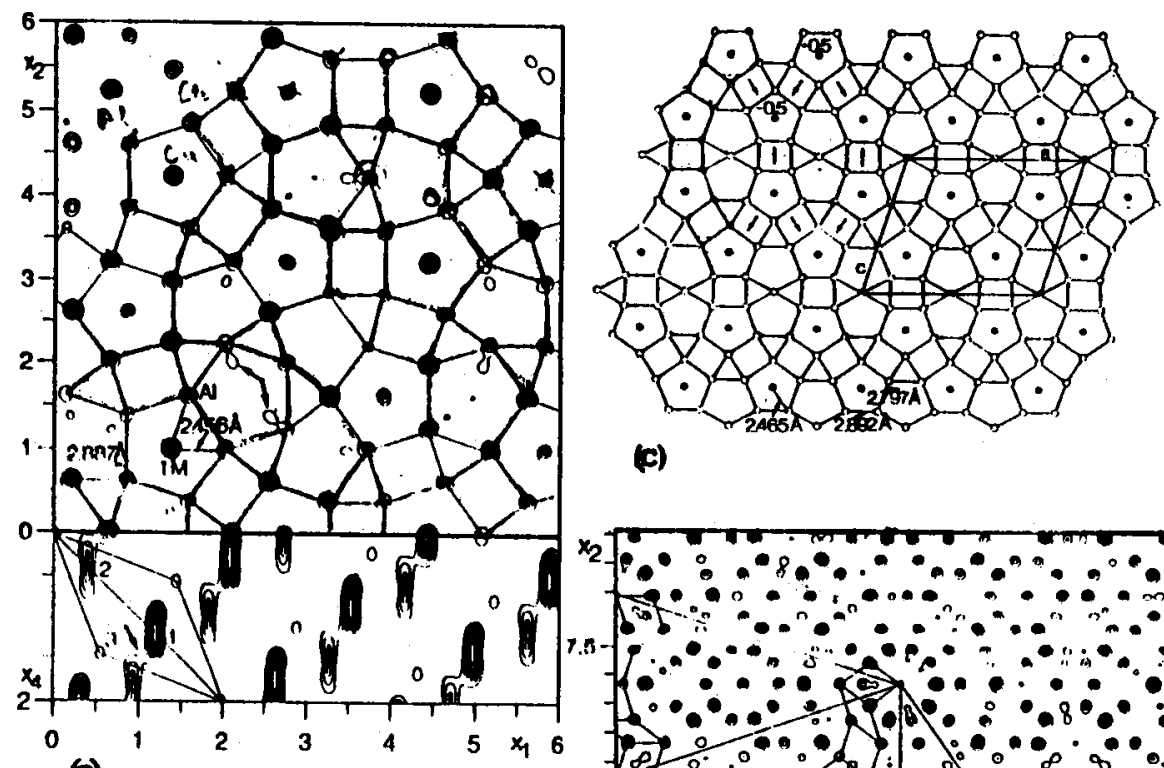

(c)

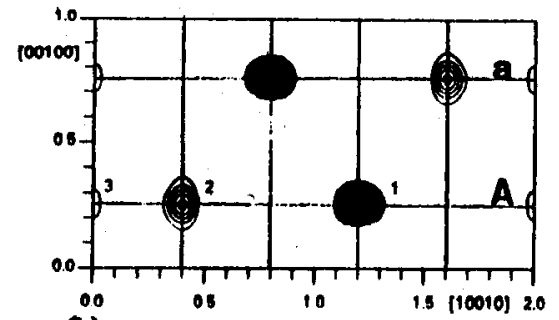

(b)

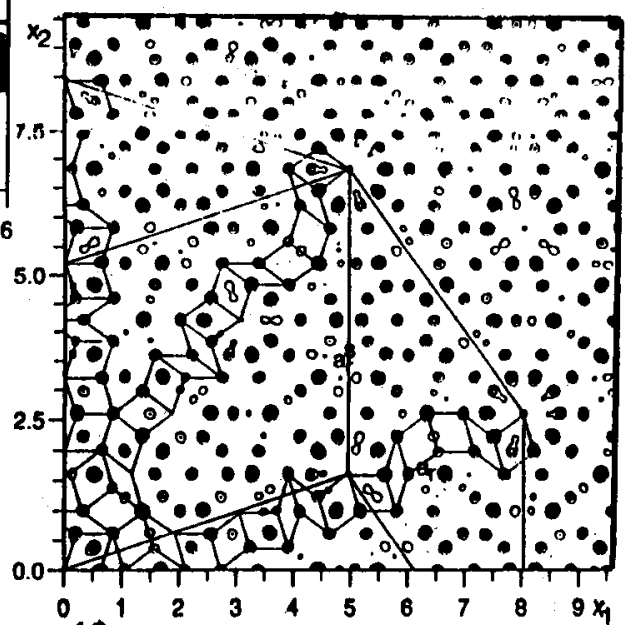

(d)

Figure 4. (a) Physical space (11000)-section of the $5 \mathrm{dim}$ electron density function of decagonal $\mathrm{Al}_{70} \mathrm{Co}_{15} \mathrm{Ni}_{15}$ together with the (10010) section to visualize the generation of real atoms from the hyperatoms market 1,2 and 3. (b) Characteristic (10110) section: the hyperatoms 1 and 2 generate an atomic layer $A$ separated by $2 \mathrm{~A}$ from the symmetrically equivalent layer a generated by $10^{5}$-screw axis parallel to [00100]. (c) Schematic drawing of the puckered atomic layer of the monoclinic approximant $\mathrm{Al}_{13} \mathrm{Co}_{4}$ with the same pentagon-rectangle strips as found in the decagonal phase. (d) (11000)-section demonstrating the relationship between small and large Penrose rhombs. 

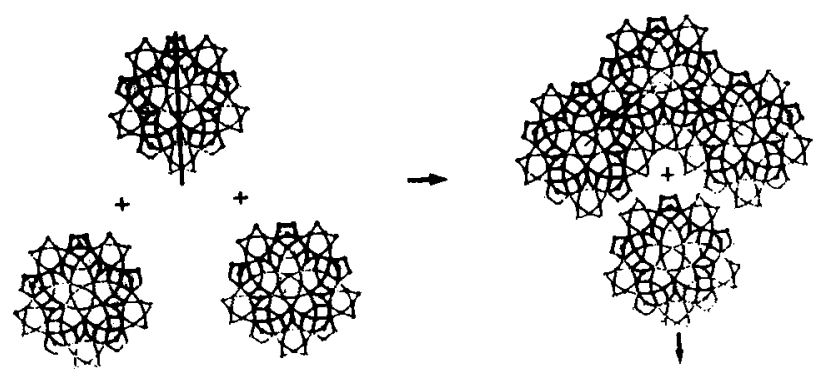

(a)

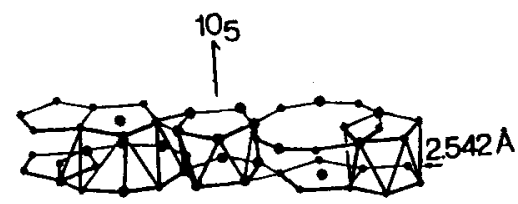

(c)

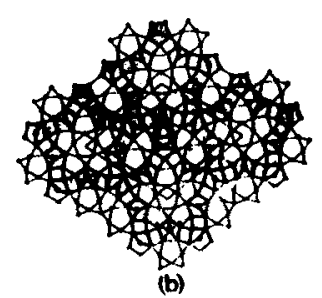

Figure 5. (a), (b) Schematic drawings illustrating how the $=20 \mathrm{~A}$ columnar clusters (shown in sections) agglomerate to a fat decorated Penrose unit rhomb and (c) stacking principle of the layers $A$ and a along the line drawn in the uppermost section of (a).

magnetic field) intelligible. The two approaches which seem to be most studied and are expected to converge closely to the experimentally observed electronic behaviour of quasicrystals correspond to (a) $\sigma-T$ and related variation governed by the quantum interference effects (weak localization and electron-electron interactions) and (b) $\sigma-T$ and other related variation controlled by power law localization of electronic states giving rise to the variation of conductivity $\Delta \sigma \propto T^{\mathrm{a}}$. In the following we proceed to describe our results on electronic conductivity variations with temperature on $\mathrm{Al}_{65} \mathrm{Cu}_{211+x} \mathrm{Ru}_{15-x}(x=2$, $1,0$ and -1$)$ qc phases.

Some of the earlier reports on the measurements of transport properties carried out on previously known quasicrystal were not conclusive and were found to be similar to either their corresponding crystalline or amorphous counterparts. Afterwards, it was realized that the real effect of the quasiperiodicity was seriously masked by their native content of structural disorder viz. the phason disorder and the presence of secondary crystalline phases. Because of their thermodynamically metastable nature, it was not possible to eliminate completely these poisoning features by thermal annealing treatment. However, with the discovery of the thermodynamically stable quasicrystalline systems e.g. Al-Cu( $\mathrm{Fe}, \mathrm{Ru}, \mathrm{Os})$ and $\mathrm{Al}-\mathrm{Pd}-(\mathrm{Mn}, \mathrm{Re})$ it has become possible to obtain highly structurally ordered quasicrystals without a contaminating crystalline phase and to analyse the effect of long-range quasiperiodic order as well as the icosahedral symmetry on the electronic transport behaviour. Recent measurements clearly demonstrate that these icosahedral alloys exhibit some unusual behaviour.

Their most characteristic features are the very high value of the electrical resistivity (up to $0.03 \Omega \mathrm{cm}$ in $\mathrm{Al}-\mathrm{Cu}-\mathrm{Ru}$ at $4 \mathrm{~K}$ and up to the record high volume of $20 \Omega \mathrm{cm}$ in Al-Pd-Re at $0.45 \mathrm{~K}$ ). The temperature coefficients of resistivity have been found to be generally negative. In case of icosahedral Al-Pd-Re alloy, the conductivity values have been found to be three orders of magnitude lower than the Mott's minimum conductivity $\left(\sigma \sim 200 \Omega^{-1} \mathrm{~cm}^{-1}\right)$ for the metal-insulator transition i.e. it lies in the conductivity range for doped semiconductors. The resistivity of these icosahedral alloys depends on the structural quality of the sample used in an unusual way; the ordered version of the quasicrystal is more resistive compared with the disordered versions of the same alloy-a feature contrary to the crystalline case. Also, the resistivity is very sensitive to sample composition.

The reason for the occurrence of high electrical resistivity in these alloys has been attributed to a pseudogap in the density of states (DOS) at the Fermi level. The occurrence of the pseudogap has been attributed to an interaction of the pseudo Brillouin zone with the Fermisurface. Recent electronic band-structure calculation for the lowest-order approximants like $1 / 1 \mathrm{Al}-\mathrm{Li}-\mathrm{Cu}$ have shown the presence of pseudogap close to the Fermi level $E_{\mathrm{F}}$. The experimental evidence for the pseudogap has also been obtained by spectroscopic measurements like $\mathrm{X}$-ray photoemission spectroscopy (XPS) and soft X-ray spectroscopy (SXS). Low temperature $(<20 \mathrm{~K})$ electronic property measurements carried out on these stable quasicrystals have invariably shown the presence of enhanced quantum interference effects (QIEs). The $\sigma-T$ data have been well fitted on the basis of weak localization (WL) effects (including strong spin-orbit scattering effects) even beyond the perturbative limit $\left(K_{\mathrm{f}} l\right)^{-\mathrm{i}}>1$. The alternative mechanism which claims to explain the electronic transport properties of quasicrystals, is based on an internal structural model which assumes the presence of the conductive $i$ blocks which are enveloped by an insulating layered structure network. For this model the electrical conduction occurs via tunneling. An alternative approach for explaining the observed variation in conductivity with temperature of a stable qc system is in terms of power law-localized electronic states leading to a power law variation in $\sigma$ typified by $\sigma \propto T^{u}$. The variation of electrical resistivity $\rho$ with temperature $T$ for $\mathrm{Al}-\mathrm{Cu}-\mathrm{Ru}$ has been measured in the low $(4 \mathrm{~K}-300 \mathrm{~K})$ as well as high temperature $(300-680 \mathrm{~K})$ ranges. In both ranges of temperature coefficient of resistance has been found to be negative. In the lower temperature range $\sigma-T$ data have been analysed in terms of the power law localization of electronic states giving rise to the powerlaw dependence of conductivity on temperature. The effect of small concentration change in $\mathrm{Ru}$ for the $\mathrm{Al}_{65} \mathrm{Cu}_{21+x} \mathrm{Ru}_{15-x}$ on its conductivity $\sigma$ and the $\sigma-T$ 
variation in the temperature range $15-300 \mathrm{~K}$ and in some cases down to $4.2 \mathrm{~K}$ have been investigated.

\subsection{Variation of electrical resistivity in the temperature range $4 \cdot 2-300 \mathrm{~K}$}

Since the electrical transport properties at lower temperatures are generally discussed in terms of conductivity $(\sigma)$ which is inverse of electrical resistivity, we present the results in terms of $\sigma$. The observations of the $\sigma-T$ variations of different quasicrystalline samples of various compositions with regard to $\mathrm{Ru}$ have been summarized in figure 6 .

The main features of the $\sigma-T$ curves are: (i) the conductivity increases with increasing temperature and (ii) the overall slopes of different curves increase with increasing $\mathrm{Ru}$ content from 13 to 15 at\% and at 16 at\% there is slight decrease in the net slope.

One significant observation of the $\sigma-T$ variation is that the ratio $R=\sigma(300 \mathrm{~K}) / \sigma(15 \mathrm{~K})=3.5$ for $\mathrm{Al}_{65} \mathrm{Cu}_{20} \mathrm{Ru}_{15}$ which is apparently much larger than any corresponding estimate for disordered metallic systems. A typical value of $R$ for disordered metallic state is 0.05 . In a recently discovered AlPdRe quasicrystalline alloy the value of $R=\sigma(300 \mathrm{~K}) / \sigma(4 \mathrm{~K})$ is as high as 190 . Another striking feature of the present alloy system is that the value of $R$ is different for different alloy systems.

The $\sigma-T$ behaviour of stable quasicrystals in most of the cases has been explained in terms of quantum interference effects (QIEs) which include the electronelectron interaction (EEI) effect and weak localization

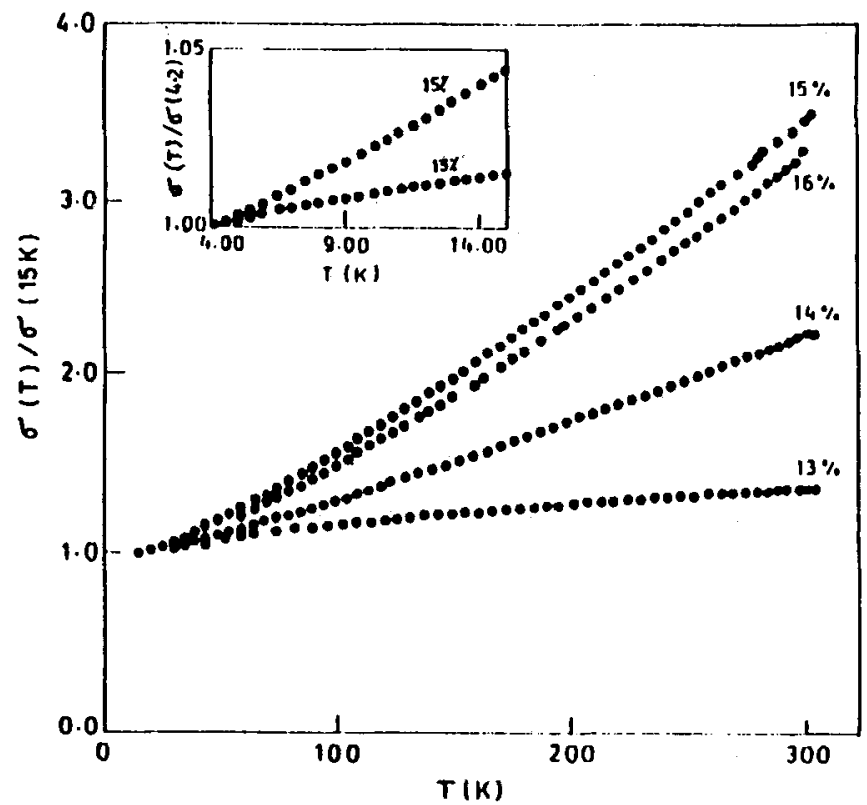

Figure 6. Curves of the normalized conductivity $\sigma$ versus $T$ for $\mathrm{Al}_{65} \mathrm{Cu}_{20+x} \mathrm{Ru}_{15-x}(x=2,1,0$ and -1$) \mathrm{qc}$ alloys. The inset shows the normalized conductivity versus temperature variation for $x=2$ and 0 in the temperature range $4 \cdot 2-15 \mathrm{~K}$.
(WL) effect. However, there are several crucial aspects which are suggestive of the fact that QIEs may not be operating for qc alloys. For example $\left(K_{\mathrm{t}} l\right)^{-1}>1$ for all stable icosahedral quasicrystals; for these conditions, the QIEs are not expected to hold good. Furthermore, since the EEI and WL are perturbative effects, the change in conductivity should be much smaller than the Boltzman constant. However, the change in conductivity has been found to be comparable with the Boltzman conductivity. Thus, the electronic characteristics of these quasicrystals appear to be beyond the regime of the perturbative effects and the conductivity variation with temperature can not be explained on the basis of concepts advanced for disordered metallic and insulating systems.

We have tried to explore alternative mechanisms which are based on electronic characteristics developed in recent years specially for qc systems. As we have pointed out earlier the nature of electronic states in case of quasicrystals has been shown to be critical. If this is valid, the conductivity is expected to vary according to a simple power law $\sigma \propto T^{\text {a }}$. A power law variation of conductivity, viz.

$$
\sigma(T)=\sigma(0)+C T^{\mathrm{a}}
$$

with temperature for $\mathrm{Al}_{65} \mathrm{Cu}_{22} \mathrm{Ru}_{13}$ is shown in figure 2 . Very strong evidence for the applicability of the power law in the case of $\mathrm{Al}-\mathrm{Cu}-\mathrm{Ru}$ system is borne out by the fact that this gives quite a good fit throughout the investigated temperature range, $15 \mathrm{~K}<T<300 \mathrm{~K}$. The power-law fitting has been obtained for $\mathrm{AI}-\mathrm{Cu}-\mathrm{Ru}$ with

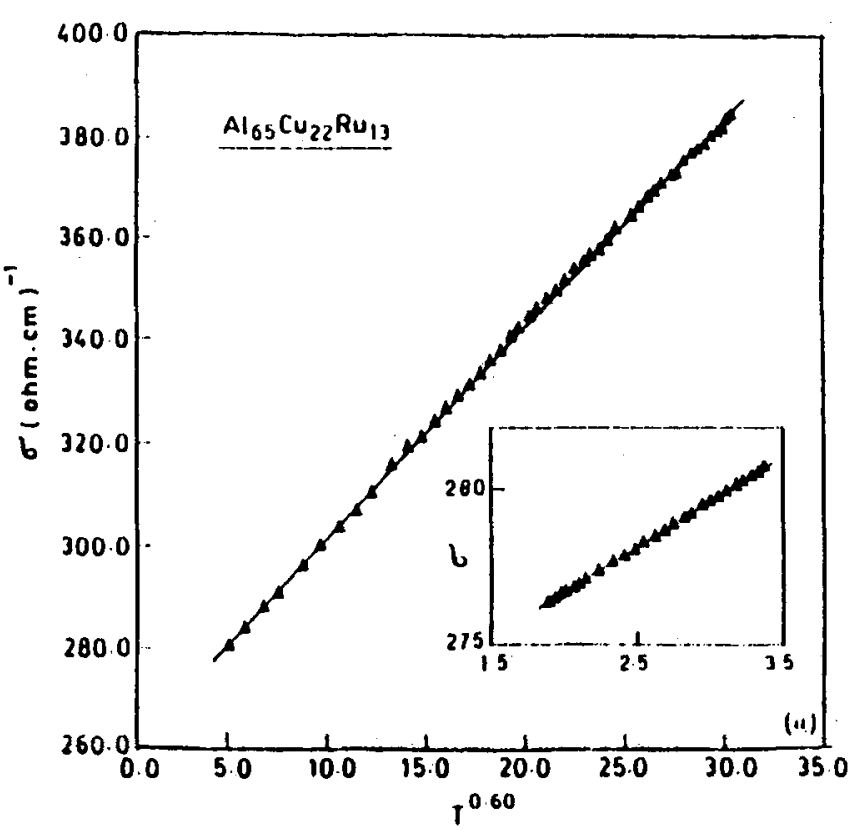

Figure 7. Curve representing the power-law variation in conductivity, $\sigma(T)=\sigma(0)+C T^{\mathrm{a}}$ for $\mathrm{Al}_{65} \mathrm{Cu}_{22} \mathrm{Ru}_{13}$. 
other $\mathrm{Ru}$ concentrations as well. The low temperature (about $4.2 \mathrm{~K}$ ) $\sigma-T$ variation is in accordance with the same power law applicable in the temperature range $15-300 \mathrm{~K}$. From the $\sigma-T$ curves in figure 7 it is noticed that $\sigma(0)$ is always finite, indicative of the fact that the $\sigma(T)$ variation does not follow a pure power law i.e. $\sigma(T)=C T^{\text {a }}$, which is expected from the theory. A detailed discussion about the origin of $\sigma(0)$ has been presented by us earlier (Lalla et al 1995).

Our results on evaluation of electronic characteristics of $\mathrm{Al}-\mathrm{Cu}-\mathrm{Ru}$ quasicrystals suggest that $\mathrm{Al}-\mathrm{Cu}-\mathrm{Ru}$, qc material which is only nearly perfect but containing some density of phason disorder as manifested by a correlation length less than $100 \AA$, the $\sigma-T$ variations at low temperatures, fit reasonably well with models taking into account QIEs. Thus for the temperature regime $(1.5 \mathrm{~K}<T<20 \mathrm{~K})$ the variation in $\sigma$ with $T$ has been shown to be in accordance with EEI i.e. $\sigma$ has been found to be proportional to $\sqrt{T}$. At a comparatively high temperature $(10 \mathrm{~K}<T<30 \mathrm{~K})$, the $\sigma-T$ behaviour has been made intelligible in terms of $\mathrm{WL}$, where $\sigma$ varies linearly with temperature. In spite of these good fits obtained for $\sigma-T$, it should be mentioned that for the stable qc $\left(K_{\mathrm{t}} l\right)^{-1}$ is usually 2 . For these conditions, the QIE are not expected to hold good.

As described earlier for $\mathrm{Al}-\mathrm{Cu}-\mathrm{Ru}$ qc materials showing good perfection (nearly phason disorder free) having correlation length $>1000 \AA$, the $\sigma-T$ fit is obtained based on power law localization of electronic states (see figures 6 and 7) leading to $\sigma-T=\sigma(0)+C T^{\text {a }}$. One remarkable feature of this fit is that it spans the whole temperature range e.g. $1.5 \mathrm{~K}<T<630 \mathrm{~K}$.

The above results suggest that (a) the different conductivity-temperature variations observed for the same qc material (e.g. besides the results on $\mathrm{Al}-\mathrm{Cu}-\mathrm{Ru}$ obtained by us as in above, the $\sigma-T$ variations as reported by Bianchi et al (1997) and Ahlgren et al (1997) for qc material Al-Pd-Re, are found to be at variance) may be due to different states of perfection (low phason density, C. $1 .<1000 \AA$, to negligible phason density, C.l. > 1000 $\AA$ ) and (b) for quasicrystalline materials the $\sigma-T$ variations embodying true qc features (aperiodicity, and self similarity, correlation length $>1000 \AA$ ) in the entire temperature region $1.5 \mathrm{~K}<T<300 \mathrm{~K}$ can be satisfactorily explained based on the concept of critical electronic states leading to power law variation of conductivity on temperature $\left(\sigma=\sigma(0)+C T^{\text {a }}\right)$. Efforts are also being made to measure electronic behaviour in stable $\mathrm{Ti}-\mathrm{Zr}-\mathrm{Ni}$ quasicrystalline alloy.

\section{Acknowledgements}

The authors are grateful to Prof. A L Mackay, Prof. G Thummes, Prof. S Ranganathan, Prof. T V Ramakrishnan, Prof. E S Raja Gopal, Prof. A R Verma, Prof. R P Rastogi for useful discussion and encouragement. One of us (MAS) is grateful to CSIR for Senior Research Fellowship.

\section{References}

Ahlgren M, Gignoux C, Rodmar M, Berger C and Rapp O 1997 Proc. of int. conf. on quasicrystal in Yamado conf. XLVII (eds) S Takeuchi and T Fujiwara (Singapore: World Scientific)

Bianchi A D, Bommell F, Chernikov M A, Gubler U, Degiorgi L and Ott H R 1997 Phys. Rev. B55 5730

Lalla N P, Tiwari R S, Srivastava O N, Schnell B and Thummes G 1995 Z. Fur. Phys. B99 43

Mukhopadhya N K, Weatherly G C, Lloyd D J and Embury J D 1993 J. Non-Cryst. Solids 63153

Pierce F S, Poon S J and Guo Q 1992 Science 261737

Shaz M A, Lalla N P, Mandal R K and Srivastava O N 1997 Phys. Rev. B55 8136

Shechtman D, Blech I, Gratias D and Cahn J W 1984 Phys. Rev. Lett. 531951

Steurer W 1994 Proc. of int. conf. on aperiodic crystals in Aperiodic 94 (eds) G Chapuis and W Paciorek (Singapore: World Scientific)

Tsuda K, Saitoh K, Terauchi M, Tanaka M, Tsai A P, Inoue $A$ and Masumoto T 1997 Proc. of the int. conf. on quasicrystal in Yamado conf. XLVII (eds) S Takeuchi and T Fujiwara (Singapore: World Scientific)

Wang C R, Lin C R, Lin S T and Chen Y Y 1997 Proc. of int. conf. on quasicrystal in Yamado conf. XLVII (eds) $\mathrm{S}$ Takeuchi and T Fujiwara (Singapore: World Scientific)

Zhang X, Stroud R M, Libbert J L and Kelton K F 1994 Philos. Mag. 70927 